\title{
LA RECONSTRUCTION DE L'IMAGE DU CORPS CHEZ LÉO, ENFANT TRISOMIQUE PRÉSENTANT DES RETRAITS AUTISTIQUES
}

Chantal Lheureux-Davidse

\section{ERES | «Cliniques méditerranéennes »}

2004/1 nº 69 | pages 289 à 308

ISSN 0762-7491

ISBN 2-7492-0269-8

Article disponible en ligne à l'adresse :

http://www.cairn.info/revue-cliniques-mediterraneennes-2004-1-page-289.htm

\section{Pour citer cet article :}

Chantal Lheureux-Davidse, «La reconstruction de l'image du corps chez Léo, enfant trisomique présentant des retraits autistiques », Cliniques méditerranéennes 2004/1 $\left(n^{0} 69\right)$, p. 289-308.

DOI $10.3917 / \mathrm{cm} .069 .0289$

Distribution électronique Cairn.info pour ERES.

(C) ERES. Tous droits réservés pour tous pays.

La reproduction ou représentation de cet article, notamment par photocopie, n'est autorisée que dans les limites des conditions générales d'utilisation du site ou, le cas échéant, des conditions générales de la licence souscrite par votre établissement. Toute autre reproduction ou représentation, en tout ou partie, sous quelque forme et de quelque manière que ce soit, est interdite sauf accord préalable et écrit de l'éditeur, en dehors des cas prévus par la législation en vigueur en France. Il est précisé que son stockage dans une base de données est également interdit. 


\section{Chantal Lheureux-Davidse}

\section{La reconstruction de l'image du corps chez Léo, enfant trisomique présentant des retraits autistiques}

Les handicaps physiques génétiques au caractère irréversible n'excluent pas des troubles psychiques associés. Ces derniers sont parfois aménageables et peuvent évoluer à la faveur d'un suivi en psychothérapie analytique.

Le suivi du jeune Léo, 15 ans, porteur d'une trisomie associée à des retraits autistiques a mis en valeur combien le bébé handicapé de naissance est confronté de façon trop précoce à une relation ambivalente de la part de son entourage, qui nuit à l'installation d'un bon pare-excitation et à la construction d'une image du corps stable.

À la naissance, tout bébé est privé du contenant utérin rythmé en continu par les battements cardiaques et la respiration maternelle. Les premières relations d'échange entre le bébé et la mère par le regard, l'attention, le holding, et les paroles prennent normalement le relais du bain utérin contenant.

Le bébé handicapé est soumis à la discontinuité des investissements dus à une ambivalence trop précoce dans la relation qui peut faire rupture et qui le plonge dans des sentiments d'être laissé tomber. Cela nuit à l'installation d'un contenant relationnel de sécurité et empêche la constitution d'un moi corporel qui tienne. Ainsi le bébé peut s'aménager dans des retraits autistiques pour se protéger des ruptures d'investissement trop éprouvantes tant que son pare-excitation n'est pas constitué.

Le travail thérapeutique avec Léo, par la nomination de son vécu sensoriel, a ouvert l'accès à la figuration des sentiments de laisser-tomber. Je déve- 
lopperai comment les phases de reconstruction de son image du corps ont pu se rejouer dans le transfert, en passant par le recours à la douleur par des automutilations. Puis je montrerai en quoi un accordage rythmique a pu relancer des possibilités relationnelles auxquelles il avait renoncé.

Léo est un garçon de 15 ans, au visage enfantin, petit pour son âge. Il est atteint d'une trisomie. Il présente une allure autistique partielle, avec des évitements de la relation et du regard. Il semble isolé dans son monde. Il se replie dès qu'il sent un conflit et pleure. Il ne joue pas avec les autres. Il aime cependant jouer aux fléchettes, ce qui l'aide sans doute à se recentrer. Il n'a pas encore manifesté de capacités d'imitation. Quand il doit traverser une rue, il reste bloqué sur le passage clouté comme figé dans son corps. La plupart du temps il reste immobile dans son corps avec un visage peu expressif. Sa démarche semble automatique, bien qu'un peu aérienne. Il ne sent pas le mouvement d'un groupe dont il fait partie quand il se déplace. Il aime les manèges pour tournoyer. Mais il s'inquiète à chaque sortie, et reste à l'écart de son groupe. Il s'inquiète de savoir où il faut aller et quand il va rentrer. Il ne se repère pas dans le temps, mais il a cependant acquis des repères spatiaux. Son langage est réduit à quelques mots qu'il répète de façon stéréotypée : " Matin, pleut, après où ? » Il ne sait pas reconnaître son prénom. Il appelle tout le temps quelqu'un et a tendance à parler tout seul. Les apprentissages sont pour l'instant très réduits. Il sait compter au maximum jusqu'à trois. Dans son atelier qui favorise le maniement des outils pour le travail du bois, il n'est pas intéressé par les activités proposées et il s'assoit souvent par terre, caché sous son établi.

J'apprends par son éducateur et l'infirmière qu'il se laisse tomber par terre quand celle-ci passe, dans une mise en scène d'angoisses d'abandon et d'effondrement psychique, puis il se cogne l'arrière de la tête jusqu'à se blesser.

Un suivi psychothérapeutique en individuel est mis en place. Léo se sent concerné par cette proposition et il viendra toujours avec plaisir à ses séances.

\section{L'ARRIÈRE TÊTE CASSÉE}

Pendant tout le trajet, Léo marche à côté de moi, il me lance quelques regards fugitifs périphériques, et se plaint d'un mal de tête en touchant sa nuque : "mal tête, cassé, mourir ». Il semble en fait content de manifester sa douleur et il vient avec plaisir. Arrivé dans le bureau, il s'assoit et voudrait que je regarde sa tête comme pour se faire ausculter par l'infirmière qui a examiné ses blessures à l'arrière de la tête à plusieurs reprises. Je lui explique le cadre de nos rencontres, et ma fonction non pas d'infirmière mais de psy- 
chologue, puis je lui propose de m'expliquer ce qui est difficile pour lui au point de se faire mal comme il l'a fait, et « ce qu'il ressent dans sa tête ».

Je tente de requalifier ce qu'il sent, même si ses affects sont pénibles et ses sensations terrifiantes. Je commence à accueillir puis à mettre des mots sur sa plainte somatique que je considère comme une difficulté à élaborer quelque chose d'impensable, sans doute d'ordre traumatique, qui ne peut pas être contenu dans une tête «bien fermée ». Je regarde donc son " arrière tête » et je nomme les sensations telles que j'imagine qu'il les ressent, d'après ce qu'il me dit et en référence à certains points théoriques développés par Geneviève Haag ${ }^{1}$. Sa difficulté à installer son regard, en association avec le ressenti d'un arrière-plan troué qu'il met en scène en s'auto mutilant l'arrière nuque et avec ses laisser-tomber devant l'infirmière, me laissent penser que le regard de confiance entre lui et les autres n'a pu se constituer. En se lançant dans le regard de l'autre, sans doute risque-t-il de s'y perdre et de tomber dans un fond des yeux pas encore bien fermé. Le regard qu'il lance ne peut pas encore faire boucle tant que le fond des yeux de l'autre n'est pas suffisamment ressenti comme contenant pour qu'il s'y retrouve.

\section{L'INSTALLATION DU REGARD}

Dans le développement normal d'un bébé, l'accroche du regard avec sa mère se constitue lorsque les regards se croisent dans l'émerveillement, avec la sensation de sécurité d'un arrière plan associé au fond des yeux de la mère, dès lors que le bébé ressent la douceur et la solidité qui contient son dos et l'arrière de sa tête lorsque sa mère le soutient en même temps qu'elle lui parle.

Quand le bébé qui vient de naître cherche du regard les yeux de sa mère, il se lance dans le regard comme s'il se lançait entièrement dans les yeux de sa mère. Le fond des yeux de sa mère est-il suffisamment solide pour permettre de renvoyer en boucle au bébé ce qu'il a lancé ? Si l'accordage entre la mère et le bébé n'a pas pu se faire pour différentes raisons (dépression maternelle, absence du père, mère absente psychiquement même si elle est présente et attentive dans ses gestes, réactivation de sa propre enfance carencée affectivement, difficulté à reconnaître son enfant par rapport à l'image qu'elle s'en était faite auparavant, difficulté d'accordage du bébé à sa mère, difficulté à voir

1. G. Haag, «Aux sources de la vie. Le langage préverbal et l'émergence des représentations du corps en situation psychanalytique individuelle ou groupale avec des enfants autistes ", Actes de langage. Quand parler c'est agir, Dialogue, $\mathrm{n}^{\circ} 123$, Paris, ${ }^{\mathrm{er}}$ trimestre 1994, p. 40-58. « Le moi corporel commence par un premier sentiment d'enveloppe qui construit plus particulièrement avec l'intégration d'un tactile et surtout d'un tactile du contact du dos allié à la pénétrance du regard. » 
et accepter le handicap de son bébé, etc.), le bébé est alors aux prises avec des angoisses primitives de détresse, que Winnicott a appelé des agonies primitives, des craintes d'effondrement, de chute sans fin, de liquéfaction, de vidage, d'effraction, de morcellement, bref de risque d'anéantissement. En lançant son regard, le bébé risque alors de partir entièrement et littéralement avec son regard, et de tomber dans un gouffre sans fond, derrière la tête de la mère qu'il vit comme béante, non fermée, comme si les pensées ou la tête de la mère ne pouvaient pas contenir l'enfant. Ce dernier s'enferme alors dans un retrait avec évitement du regard, dans un recours à une forme de survie psychique. Il ressent du même coup son propre dos et le fond de ses yeux dans son arrière-plan comme également troués.

\section{UNE RELATION QUI TIENNE}

Je dis à Léo qu'il a sans doute peur que sa tête ne soit pas bien fermée quand il me regarde de façon fugitive et que son coté gauche n'est pas bien accroché à sa droite, tandis que je me tiens à ses côtés. Cela fait-il écho à son inquiétude concernant notre relation qui ne serait pas encore bien " accrochée » ? Je lui demande si en se tapant la tête en arrière il n'avait pas peur que sa tête ne soit pas bien fermée, surtout si « je ne m'occupe pas bien de lui dans ma tête ». Lorsqu'un enfant pense que sa tête n'est pas bien fermée, il oublie qu'il a un dos et un arrière de la tête. Léo est très attentif à mes interprétations et semble être apaisé, au point que son regard " $s^{\prime}$ installe » avec sécurité dans ma direction pendant qu'il acquiesce à mes propos, d'un « oui » prononcé du bout des lèvres.

\section{LA FONCTION DE LA DOULEUR DANS LES AUTOMUTILATIONS}

Je m'interroge sur le recours à la douleur par le biais du cognement de tête et l'automutilation. Je comprendrai ces automutilations selon deux perspectives.

Si le recours sensoriel douloureux aide à la figuration de vécus impensables, tels que "s'être senti désinvesti ou laissé tomber ", sur le chemin de la représentation d'un arrière-plan cassé ou défaillant - arrière-plan de sécurité qui se constitue normalement avec la mise en place du regard - , il tente de casser et d'annuler des sensations corporelles à cet endroit, dans le sens d'une décharge d'intensité trop forte. Cela provoque des vécus terrifiants de risques d'anéantissement, des angoisses de « laisser tomber » ou de « vidage par l'arrière-plan béant » et entraîne par voie de conséquence la perte des limites du moi corporel et de l'altérité. 
La perspective de survie à ces angoisses induit une recherche de retrouvailles de sensations afin de se sentir exister dans son corps et dans sa tête, et de tenter d'installer un sentiment de continuité d'existence selon l'expression de Winnicott.

La douleur des automutilations pourrait être comprise à travers une deuxième perspective, comme un passage efficace pour accéder à des sensations perdues. Ce recours à des sensations fortes assurerait une réintégration sensorielle de zones sensoriellement effacées. L'intensité de la violence des automutilations me semble être à la mesure de la difficulté à contacter à nouveau ses sensations. En insistant, la réanimation sensorielle commence à poindre, quand la douleur finit par s'installer en continu. "Se sentir mal » prend alors tout son sens pour éviter de ne plus se sentir du tout. Cette réassurance de son existence par le ressenti corporel participe à l'atténuation des angoisses d'anéantissement.

Cependant, sentir à nouveau son corps, non pas dans un plaisir contenant de douceur et de fermeté, mais dans la douleur entraîne rapidement une saturation sensorielle ingérable, qui oblige à annuler les perceptions du corps si l'intensité en est trop forte, contrairement au but recherché.

\section{DE LA DOULEUR AUX ÉMOTIONS FORTES PARTAGÉES}

L'accompagnement thérapeutique consistant à qualifier ces ressentis douloureux et à nommer les parties clivées, peut contribuer à la prise de conscience pour l'enfant de ses propres ressentis, puis à la contenance des sensations de débordement. Le partage émotionnel intense dans ces moments interprétatifs transforme les sensations intenses de douleurs en émotions fortes partagées, et ouvre l'accès au sentiment d'altérité.

Je pense que l'intensité émotionnelle partagée dans le cadre thérapeutique est indispensable pour déplacer les sensations fortes de douleur qui isolent l'enfant. En effet, après une perte de sensations trop prolongée, l'accès à la douceur ne peut se produire qu'à la faveur d'un passage par des sensations fermes et fortes. C'est ainsi que le recours à la douleur par les automutilations est parfois le passage obligé chez certains enfants à composantes autistiques, pour pouvoir paradoxalement accéder à des ressentis plus doux, et ceci tant que le sentiment d'altérité n'est pas accessible.

Dans des aménagements plus heureux et moins mortifères, je pense que les stéréotypies visant à obtenir des émotions esthétiques, comme les jeux avec la lumière ou les reflets, ou comme les émotions sonores procurées par la musicalité de la voix, permettent d'accéder aux ressentis corporels sans passer par la douleur. 
Cependant, il me paraît important de décoder au mieux le mode d'aménagement des sensations fortes créé par l'enfant, qu'il s'agisse par exemple de la douleur, des automutilations, de l'extase, de l'autosensualité ou des jeux de lumière, afin qu'il accède à la conscience de ses ressentis corporels. En l'y rejoignant par la nomination de ces ressentis, une expérience de rencontre se crée en rendant possible la réintégration du moi corporel.

Si le partage émotionnel se situe souvent hors des activités ordinaires et hors contexte social, il facilite la curiosité et l'intérêt de l'enfant pour l'échange, qu'il recherchera par la suite, même dans un contexte plus socialisé.

Dans un second temps, l'interdit de se faire mal peut être alors plus opérant. Après la réintégration sensorielle par la douleur, la proposition d'un relais sensoriel plus doux peut être entendue, ne serait-ce que par le réceptacle engendré par l'écoute du thérapeute, par l'enveloppement de la musicalité de sa voix, de ses pensées qui font sens et par son regard nommant les parties démantelées, dans le sens du démantèlement sensoriel réversible dont parle Meltzer 2 .

Devant Léo je me réjouis du plaisir qu'il éprouve à l'occasion de retrouvailles avec des sensations de parties clivées, et il jubile également face à cet émerveillement qu'il peut rapidement s'approprier à travers cette prise de conscience de ses ressentis ainsi que le partage avec moi.

Je lui dis alors que lorsqu'il a mal à la tête, il est sûr d'avoir une tête, mais qu'il n'a pas le droit de se faire mal. Avant d'interdire à un enfant de se cogner la tête, il est nécessaire de reconnaître pourquoi il a besoin de s'automutiler. Dans un second temps l'enfant peut entendre plus facilement l'interdit de détruire son corps, et la proposition d'une autre solution pour continuer à sentir son dos et sa tête, mais cette fois-ci avec douceur et fermeté.

\section{LA RÉANIMATION SENSORIELLE DES ZONES CORPORELLES CLIVÉES}

Dès la séance suivante, Léo ne se cogne plus la tête. Le long du trajet il me rappelle que durant le trajet précédent, il s'était plaint de mal de tête. Je sens cette fois ci qu'il ne s'agit plus d'une figuration d'un traumatisme mais d'une évocation, dans le sens d'une représentation en voie de symbolisation.

Léo apparaît moins angoissé, il sourit en me parlant de «tête, mal », et je retrace en effet nos discussions autour de ce thème. Semblant assuré avec sérénité de la fermeture de sa tête et de son arrière-plan, il peut me regarder

2. D. Meltzer, J. Bremner, S. Hoxter, D. Weddell, (1975), Explorations in Autism, London, the Roland Harris Educational Trust, Clunie Press, trad. Fr. G. Haag, Explorations dans le monde de l'autisme : étude psychanalytique, Paris, Payot, coll. «Science de l'homme », 1980, 266 p. 
un peu plus longuement en souriant et il se met alors avec assurance à attraper son nez. Il le tire si violemment pendant tout le trajet en disant «nez », que je crains qu'il se l'arrache ou qu'il se le casse. Je sens qu'il n'est pas question de le lui interdire dans un premier temps. Je mesure l'intensité de sa force à mettre en place cet axe vertical jusque-là démantelé au niveau de son visage, cette fois-ci à l'avant-plan au milieu de son regard. Je prends le risque d'une cassure du cartilage de son nez, mais je sens que Léo est de plus en plus concerné sensoriellement par ce recours à la douleur comme tentative de réanimation de cette zone perdue, et je décide de lui faire confiance dans son instinct de survie et dans ses sensations retrouvées pour éviter une fracture corporelle, même si l'idée m'en est pénible.

La séance commence ainsi. Il me montre à quel point il retrouve la sensation qu'il a un nez grâce à la douleur et à la violence qu'il s'inflige.

Afin de lui permettre de reconstituer des parties de son image du corps jusque-là défaillante, je me réjouis avec lui du fait qu'il retrouve la sensation d'avoir un nez. Je lui dis dans un premier temps qu'il avait complètement oublié qu'il avait un nez, et combien «c'est formidable de le retrouver ». Afin que cela passe par une boucle transférentielle, je lui dis dans un second temps que moi aussi j'ai un nez et que lui comme moi n'avons pas le droit de nous faire mal au nez, puis que nous sommes bien certains qu'il a retrouvé son nez.

Ce déroulement lui a permis d'arrêter spontanément de se faire mal et de nommer son nez en me regardant dans les yeux avec apaisement après un instant de jubilation.

Après la reconstruction de son axe vertical tant au niveau vertébral que dans son avant-plan, il découvre chaque partie de son visage en lui faisant subir l'épreuve de la tentative d'arrachement : le front, les gencives, les dents, puis les joues, les paupières, les oreilles, la peau de ses poignets et du dessus de ses mains. Il se réjouit de retrouver chaque partie de son corps démantelé, dans une jubilation de se sentir exister en me regardant avec une sérénité extatique.

Ainsi il rassemble une image du corps jusque-là démantelée, depuis l'axe vertébral, avec l'arrière tête, le nez et le front en avant plan, puis l'accroche des deux moitiés du corps par la charnière verticale, les différentes parties du visage et les mains. Ces parties correspondent aux sensations effacées lors de ses refuges autistiques, quand il était trop absorbé dans ses pensées clivées de ses sensations corporelles, ce qui l'isolait dans sa bulle autistique, en dehors de ses bords corporels.

Les retrouvailles de Léo avec ses sensations corporelles le submergent vite, à la mesure du temps trop long pendant lequel il en était privé jusqu'alors. 
Il investit peu à peu tout son corps jusqu'aux jambes qu'il tente d'arracher sous mon regard pour en éprouver la solidité contenante, et tapote ses pieds sur le sol pour les sentir. Puis il retrouve des sensations au niveau de son sexe qu'il ne manque pas de toucher pendant les séances. Je lui dis que le fait de retrouver son corps lui fait ressentir beaucoup de choses jusque dans son sexe de garçon, mais dans un deuxième temps j'ai le sentiment de lui apprendre l'interdit de se toucher dans ses parties intimes en ma présence ou en la présence de quiconque. Je lui dis qu'il a le droit de se toucher avec plaisir quand il est tout seul. Il accepte cette règle sociale qui ne le concernait pas jusqu'à présent. Le défaut d'accès à la socialisation des enfants pris dans des retraits autistiques va de pair avec un défaut d'intériorisation et d'intégration de certaines lois, qu'il est bon de rappeler de temps en temps, ce qui rassure toujours les enfants qui n'ont aucune idée de leur jardin secret, ni des interdits fondamentaux.

\section{UN PARE-EXCITATION DÉFAILLANT}

Dans les séances suivantes je trouve que Léo est plus présent, son regard s'installe, il m'interpelle davantage. Il est davantage en contact avec ce qu'il ressent. Mais en étant plus concerné par ses sensations, il en est vite submergé, son « pare-excitation » étant encore très peu constitué. Le cadre thérapeutique passant par la médiation métaphorique de l'architecture des murs est alors éprouvé dans sa solidité par Léo lors de ses débordements. Il tente de casser tout ce qui l'entoure dans la pièce et de faire tout tomber avant de quitter la séance. La permanence de l'objet n'est pas encore constituée pour lui et il semble vouloir détruire avant de partir ce qui risque de disparaître de sa pensée en dehors du temps des séances. Ainsi il me fait éprouver pendant de nombreuses séances ses débordements émotionnels dans lesquels il essaie d'abord de m'entraîner dans une co-excitation et ses rires en cascades, pour ensuite tenter de tout casser sur son passage en retournant vers son groupe.

Si je peux me réjouir de son nouveau contact avec l'image de son corps, du plaisir de ses ressentis corporels, autant que de son accès naissant à l'altérité et de sa jubilation lors du partage émotionnel dans la relation, je me trouve dans l'impuissance à contenir ou canaliser ses débordements à chacun de ses départs ou dès que la relation s'installe dans le temps.

Je comprendrai cette attaque du cadre non pas comme une « attaque des liens » tel que Bion a pu le décrire, mais comme une tentative de maîtrise de la différenciation au moment où l'investissement le submerge. Cette attaque du cadre semble mettre en figure ses débordements émotionnels et l'éclatement de son esquisse de " pare-excitation » encore trop fragile. La tentative 
de destruction du cadre viserait, me semble-t-il, à la destruction du non-moi, dans une perspective de survie de son moi corporel tout juste récupéré. D'un point de vue économique, mieux vaut organiser la rupture du cadre plutôt que la subir de façon impromptue et catastrophique tant que sa capacité contenante n'est pas intériorisée.

Cependant le résultat de cette tentative de destruction peut l'entraîner vers une excitation qui ne cesse de s'accroître sans pouvoir atteindre la décharge du surplus de son intensité. Il convient alors de proposer une alternative pour éviter ce débordement d'excitation qui devient alors mortifère. Avec Léo le recours à un tiers permet pendant de nombreuses séances de traverser le passage difficile entre la sortie de la séance et l'arrivée dans son groupe. Je commence à ressentir toute mon incapacité à lui permettre de traverser ces moments sereinement et je guette le moment de risques de débordements émotionnels à travers quelques indices tels que des rires en cascades, ou lorsqu'il remplit un verre de dînette en le faisant déborder, afin de lui éviter un arrachement destructeur et de lui permettre de bons essais de séparation. Arrivé dans son groupe il retrouve son calme immédiatement.

Léo investit pendant quelques mois les jeux de dînette. Il oublie au début de se servir tandis qu'il est entièrement consacré à mon bien-être avant de sentir le droit à son existence. Je lui dis alors qu'il voudrait entièrement s'occuper de moi, mais que c'est trop de travail pour lui s'il oublie de s'occuper de lui, et que cela ne relève pas de ses responsabilités.

Par la suite il oubliera plus rarement de se servir. Ses jeux de dînette amorcent des mouvements de symbolisation puisqu'il fait un peu semblant de manger tout en buvant réellement et abondamment de l'eau, et qu'il met dans sa bouche, cuillère, fourchette et aliments en jouets sans pouvoir encore faire complètement semblant. Les jeux de dînette avec moi le submergent vite, bien qu'il les réclame, et je peux mesurer à quel point il est vite débordé par une intensité relationnelle à laquelle il n'est pas habitué. La bonne purée faite à la maison que lui prépare souvent sa mère pour lui faire plaisir est systématiquement servie. Dès qu'il investit dans la durée les jeux, il est vite débordé, ce qu'il met en scène en faisant déborder l'eau de son verre et en riant en cascades sans pouvoir s'arrêter, jusqu'à ce qu'il se mette à tout casser dans le bureau, attaquant le cadre, le bureau, les murs et tout ce qui se trouve sur les étagères, ainsi que tout ce qu'il voit sur son passage en repartant dans son groupe. Lorsqu'il se trouve dans cet état, il fait en sorte de m'entraîner dans une co-excitation que je dois interrompre rapidement pour qu'il ne se mette pas dans une situation où il aurait tout détruit. Pendant une vingtaine de séances il reste envahi par ces débordements émotionnels. Je fais alors appel à un tiers qu'il réclame, ce qui l'apaise immédiatement mais 
sans que cela puisse être longtemps efficace. Quand ces enfants arrivent à mettre le thérapeute dans ces états de débordement, il convient d'interrompre immédiatement cela. Le meilleur signal pour intervenir dans ce sens est d'être attentif à son contre-transfert, et de repérer ces mouvements d'emprise qui entraînent un empêchement de penser, et un dépassement des limites du cadre thérapeutique.

À la suite de quelques séances où il veut encore tout casser bien qu'il soit toujours content de venir en séance, je me sens découragée de ne pas trouver la manière de permettre à Léo de ne pas tout détruire. Ma peur de ne pas réussir à contenir ses débordements me plonge dans des mouvements dépressifs à la fin des séances avec lui.

\section{LA RECHERCHE D'UN ACCORDAGE RYTHMIQUE :}

VITESSE DE PRÉCIPITATION ET DENSITÉ, APESANTEUR ET TRANSPARENCE

Lors d'une nouvelle séance, alors que nous nous dirigeons vers le bureau, je marche en silence à côté de lui et je me laisse éprouver le bien-être que dégage à mes côtés Léo, heureux d'aller à sa séance, tout en ayant à l'esprit ses débordements à chaque fin de séance depuis plusieurs mois.

Léo semble marcher comme hors du temps. Ses mouvements de visage lorsqu'il tourne la tête pour me regarder semblent être au ralenti et marqués d'une certaine béatitude. Son visage est radieux. Il se laisse baigner avec délice par la luminosité de cette journée, le visage tourné vers le soleil. Je réalise enfin que ses tentatives de tout casser et de faire tomber les objets de la pièce dès lors qu'un investissement s'installe cependant avec bonheur, traduisent sans doute une incapacité à intégrer sensoriellement les émotions qui le submergent, et que ses mouvements au ralenti lui permettent précisément de ralentir le temps d'intégration des événements relationnels dont il est demandeur. J'ai alors le sentiment que la vie va trop vite pour lui et qu'un rythme plus lent pourrait sans doute lui permettre de continuer à être présent dans la relation sans en être submergé.

Je me décide alors à me mettre à son diapason, bien que je me sente déjà dans un rythme pourtant calme. Je commence à ralentir mes gestes et mes paroles comme dans un film au ralenti.

Je me sens alors moi aussi « comme hors du temps ", tout en étant dans l'espace de la séance marquée par le cadre temporel de sa durée. Je partage son rythme au ralenti tant dans ma façon de parler que dans mes déplacements corporels, comme en état d'apesanteur. Je constate que Léo s'en trouve immédiatement apaisé et que son implication relationnelle n'en est que meilleure, tant dans son regard que dans les mouvements de son corps. Sa participation aux échanges peut s'installer dans la durée. Je ressens alors des 
états contre-transférentiels de diminution de sensations de densité de mon corps, des états de flottement et de dilatation de mes limites corporelles dans une impression de transparence. Je suppose alors que Léo se met dans le même état pour éviter de se sentir débordé par la vitesse de précipitation au moment où il contacte à nouveau la densité de son corps, dès qu'il est aux prises avec ses sensations, ses émotions et ses sentiments partagés dans la relation thérapeutique.

Désormais Léo peut rester tout le temps de sa séance en investissant la relation sans débordement, sans plus casser ni chercher à détruire ou éprouver la solidité du cadre. Ses trajets de retour vers son groupe se passent maintenant dans un calme surprenant. Il peut investir les jeux de dînette et les pots de peinture sans être la plupart du temps submergé. Je fais en sorte que les jouets ou les pots de peinture qui lui procurent beaucoup d'émotions, passent par mes mains qui ralentissent le mouvement des objets avant qu'il les reprenne pour les installer. Cela lui permet sans doute de constituer à travers ma personne un « moi auxiliaire » ayant une fonction de " pare-excitation ».

Les ressentis corporels semblent ainsi plus acceptables pour lui. Et bien que ces échanges paraissent encore peu incarnés, ils constituent pour Léo une expérience de rencontre vécue dans la confiance et à son rythme, même si l'accès à l'ambivalence de la relation n'est pas encore au programme de nos rencontres.

C'est à partir de ce moment qu'il installe la dînette non seulement pour lui et moi mais pour Leslie, une copine de son ancien centre qu'il fréquentait quelques années auparavant. Quelques nouvelles amorces de symbolisation se mettent en place peu à peu.

Il fait alors beaucoup de progrès et il profite de l'expérience de nos rencontres pour intérioriser peu à peu le bénéfice d'émotions partagées dans un cadre contenant.

Je continue à vivre les séances au ralenti comme en état d'apesanteur pendant plusieurs mois. Et je sens que ces temps idéalisés, hors champ de l'ambivalence, sont un passage nécessaire pour accéder au ressenti corporel en continu à l'origine de la constitution du « sentiment d'exister » dont parle Winnicott.

\section{L'INSCRIPTION D'UN MOI CORPOREL NAISSANT ET SON INTÉRIORISATION}

À cette époque, il souhaite faire de la peinture, afin de laisser des traces, non pas sur la feuille qu'il me demande uniquement pour accéder aux peintures, mais sur son corps. Il feint de peindre des points avec application sur la feuille et joue à s'étonner de " déborder » tel un enfant qui déborde du cadre d'un coloriage, pour faire atterrir son pinceau plein de couleur sur les 
zones corporelles récemment retrouvées au cours de la reconstruction de son image du corps. Ainsi il marque à la peinture sa tête, son front, son nez puis ses mains et réclame que je le nettoie après chaque trait, en nommant ces parties marquées et effacées, avec un plaisir d'appropriation non dissimulé, qu'il affirme avec bonheur. Léo retrace chaque partie de son visage, il savoure l'effacement de traces que je lui offre et semble vérifier dans la glace l'intégrité de son moi corporel après chaque effacement et l'intériorisation qu'elle suppose.

J'ai le sentiment qu'il marque ainsi de traces son existence dans son corps et qu'il profite du transfert pour faciliter ces mouvements $d^{\prime}$ inscription dans l'existence. Les traces sensoriellement éprouvées dans leur marquage et dans leur effacement puis dans la vision de leur absence semblent participer à l'intégration de son moi corporel.

\section{L'EFFROI DE LA RENCONTRE AVEC LE BÉBÉ « LOUP GAROU »}

Lors d'une séance suivante, il réclame à nouveau la peinture. Je m'aperçois maintenant qu'il sait dessiner un bonhomme avec un corps-tête rond, des yeux, une bouche, deux jambes et des traits en guise de bras, témoin d'une image du corps en voie de constitution (ce qui n'était pas apparu dans les peintures précédentes). Il se présente désormais au milieu de deux personnages comme s'il avait intégré par le transfert sa place entre ses deux parents. Il me demande du regard s'il peut peindre sur l'extrémité de ses doigts. Je lui donne mon accord en ne sentant pas qu'il s'agisse d'un quelconque débordement. Il m'annonce alors son identité : «Léo, loup garou ». Léo prend de la peinture bleu clair et macule délicatement d'un point chaque extrémité de ses doigts sur les ongles, en m'expliquant que ces marques représentent les «griffes » du «loup garou ».

Il choisit ensuite du rouge et me donne le pinceau en me priant de peindre le dessus de mes mains sur toute la longueur des doigts. Il m'explique que je suis également un loup garou mais avec de grandes griffes. Je suis impressionnée par mes grandes et longues griffes rouges face à ses petites griffes au bleu de l'innocence. Il se met à retrousser son nez et plisse tout son visage en émettant un rugissement de petit lion qui est destiné à m'intimider et il me présente ses petites griffes bleu layette.

Il tente de m'entraîner dans une co-excitation au sol à quatre pattes en rugissant. Je lui indique que je ne participerai pas à son invitation et que je reste assise sur ma chaise à mon bureau. Je le perds alors de vue et je l'entends ramper à quatre pattes autour de mon bureau tel un bébé se déplaçant de la sorte pour retrouver sa mère. J'aperçois Léo par terre sur le côté du bureau levant la tête vers moi tel un nourrisson en recherche du regard lumi- 
neux de sa mère. Il me dit: "Maman, regarde! ». Je me lève pour me pencher au dessus de lui et je me vois comme au dessus d'un berceau, découvrant mon bébé qui me cherche du regard. Léo me présente ses petites griffes bleues et grogne tel un petit lion qui veut faire peur en plissant son visage dans une grimace épouvantable pour faire «le méchant » et en m'expliquant qu'il est un «loup garou ». Je me laisse impressionner par ces petites menaces qu'il vit très intensément et il me demande de montrer mes grandes griffes rouges et d'avoir un visage grimaçant de loup garou qui doit lui faire peur avec des grognements.

Nous nous confrontons en face-à-face d'assez près à jouer à nous faire peur et à nous intimider réciproquement pendant un long temps. Et je lui propose quelques colorations interprétatives pour évoquer la difficulté dans ses premiers mois d'existence à se sentir un être agréable à regarder et combien il attend autre chose de la rencontre. Cela ressemble alors à des grimaces de loup garou ou chacun se met à avoir peur de l'autre et à faire peur à son tour. Je lui demande si c'est comme cela qu'une mère découvre son petit garçon handicapé quand elle est effrayée de voir son visage qui ne correspond pas à ce qu'elle a imaginé. Il acquiesce. Je joue les parents qui découvrent avec frayeur, déni et dégoût le handicap de leur bébé, qui leur fait peur comme le loup garou que joue Léo (qui veut pourtant accrocher de son regard la relation). Ainsi je joue un regard tantôt inquiétant, tantôt agressif ou désemparé face à ce petit garçon qui me fait peur. Je lui dis combien il m'a fallu du temps pour ne plus avoir peur de lui et ne plus lui faire peur par mon regard effrayé et effrayant, et que je n'ai pas vu tout de suite qu'il ne demande qu'amour dans une relation partagée et un échange de regards lumineux et contenants que je ne peux pas encore lui donner.

Léo acquiesce au fur et à mesure à mes interprétations et à la nomination du handicap lié à sa trisomie, tout en continuant à jouer cet échange de menace, d'agressivité entre loups garous. Sans doute s'était-il identifié au miroir grimaçant et dégoûté que lui renvoyaient son entourage.

Après ces interprétations, il arrête le jeu et il réclame avec une grande sérénité que nous lavions la peinture de nos mains. Il repart tranquillement vers son groupe. Je me remets peu à peu de l'intensité émotionnelle de cette séance. Léo, quant à lui, passe un après-midi dans un calme évident et une réelle présence.

Les traumatismes sans doute à l'origine du retrait autistique par l'évitement du regard dès les premiers mois de sa vie deviennent représentables. Je me réjouis intérieurement de son accès à des mouvements d'ambivalence dans la relation sans qu'il s'en échappe, et je mesure à quel point cette représentation des traumatismes relationnels du début de sa vie ont dû rester 
impensables jusqu'à présent et comment le refus de l'ambivalence, qu'il subissait de façon trop précoce, l'en protégeait grâce à ses retraits autistiques.

\section{DU DÉNI À L'ACCEPTATION DU HANDICAP}

Par la suite, nous avons pu ré-évoquer en présence de sa mère, la peur que provoque chez les autres la vue d'un enfant handicapé et sa trisomie en particulier, et la tristesse que les autres le rejettent la plupart du temps quand ils l'identifient à sa trisomie, avant même qu'ils ne perçoivent son être en désir de relation comme est en droit de l'éprouver tout être humain.

Quand j'ai rencontré une première fois la mère de Léo en début de thérapie, je l'ai trouvée chaleureuse, attentive et laissant une autonomie suffisante à son fils. Il lui avait fallu au moins un an pour accepter le handicap de son fils à la naissance. Actuellement, Léo prend bien sa place dans la famille auprès de ses parents et de sa sœur aînée. Il commence à participer à la vie de famille et s'isole moins. Son père investit la relation avec son fils à travers des activités sportives partagées. Malgré le retrait autistique de leur fils, ses parents n'ont pas encore envisagé une orientation adaptée pour lui. Ils le pensent capable d'intégrer un CAT, alors que les apprentissages relationnels ne sont qu'esquissés. Leur investissement affectif bien installé avec leur enfant va encore de pair avec un déni des conséquences du handicap.

Pendant cet entretien, Léo explique à sa mère en la regardant toutes les étapes des retrouvailles avec son image du corps, en retraçant par ses gestes chacune des parties de son corps qu'il tire en mimant un arrachement, sans pour autant insister ni se faire mal. Sa mère ajoute que Léo est davantage présent aux autres et à lui-même à la maison. Il commence à s'impliquer dans la vie de famille et son regard s'installe de plus en plus. Il ne sait pas encore se défendre des bousculades infligées par les copains de son groupe, qui tentent de le faire réagir quand il reste trop figé dans son corps. Mais il tient maintenant sa place dans son groupe sans plus se terrer sous son établi.

\section{L'ACCÈS À L'AMBIVALENCE ET LA CONSTITUTION D'UNE DOUBLE ENVELOPPE}

Léo commence à s'affronter aux camarades de groupe hors du champ de vision de son éducateur, et suscite aussi les coups face auxquels il ne peut encore se défendre ni riposter. Ces manifestations d'agressivité sont de bonne augure, comme signes de sortie de ses comportements autistiques, bien qu'il faille être extrêmement vigilant pour éviter des conséquences fâcheuses de mise en danger des autres comme de lui-même.

Il met en scène pendant quelques séances des figurines d'animaux et un personnage musclé articulé qu'il appelle "le méchant ». L'organisation spa- 
tiale des animaux décrit un demi-cercle autour de lui-même. Il place «le méchant » au centre tout près de son axe vertical, sur la table. Les animaux placés en arc de cercle regardent "le méchant».

Je le sens au bord d'un débordement émotionnel, prêt à mettre en scène des bagarres qui mettent en danger l'espace thérapeutique. Je me sens investie en des déplacements transférentiels par les qualités des animaux qui "l'em-bêtent », de ces bêtes qu'il met en scène comme pour figurer son entourage déshumanisé qu'il a dû subir par son handicap. Je lui propose alors que chaque animal qu'il met en scène passe (par le transfert) dans mes mains. Je ralentis le mouvement des gestes, des figurines et de la parole, avant que je lui redonne et qu'il prénomme chacun d'entre eux. Cela l'apaise immédiatement. Il nomme chaque animal des prénoms choisis parmi ses camarades de l'institution et parmi le personnel encadrant. J'écris le prénom sur un papier que je scotche sur le dos de chaque animal. Chaque prénom est judicieusement choisi en fonction des qualités qu'il ressent en résonance entre l'animal et la personne dont il emprunte le prénom. Ainsi, les enfants les plus autistiques sont devenus des éléphants avec leurs grandes défenses, son éducateur devient un lion, ses éducatrices des girafes, une de ses copines nonchalante prend la figure d'un ours assis, son père qu'il nomme Papa Victor apparaît sous la forme d'un hippopotame. Il me désigne par un mouton blanc et luimême se représente à travers un rhinocéros à cornes, à la peau cuirassée.

Il réclame les jouets constitués par des maisons en bois et des arbres, et les dispose à l'extérieur pour doubler l'arc de cercle des animaux. Ce double contenant semble lui permettre une certaine sécurité spatiale qui garantit un lieu bien fermé pour accueillir les bagarres destructrices qu'il veut mettre en scène au centre avec «le méchant ».

Les bagarres peuvent commencer. Il fait entrer en scène au centre de l'arène chaque animal qu'il appelle du prénom choisi afin qu'il attaque «le méchant». Chaque animal doit avoir peur du méchant. Une fois attaqué, «le méchant » riposte en faisant virevolter l'animal. Puis l'animal s'affronte à nouveau au méchant qui tombe à terre sans se relever. L'animal reprend alors sa place calmement dans l'arc de cercle des animaux, mais cette fois-ci le dos tourné.

Les autres animaux jouent à tour de rôle le même scénario de bagarre jusqu'à ce que l'arc de cercle formé par les animaux qui tournent à présent le dos au méchant, se reconstitue. Les va-et-vient des animaux parcourent bientôt un mouvement radiaire ${ }^{3}$ en boucles qui passe par le centre. Il range

3. Geneviève Haag a développé cette notion de mouvement radiaire à partir de la figuration d'échanges de relation retrouvés par le regard chez des enfants autistes. 
ensuite chaque animal dans la caisse à jouets en les prénommant à nouveau avant de les voir disparaître dans la caisse.

Le jeu de l'affrontement semble ainsi participer à l'intériorisation de la difficulté relationnelle et à la constitution d'un dos solide qui supporte les attaques tout en les contenant. Il élabore ainsi ses capacités d'agressivité qui ont dès lors moins besoin de s'exprimer dans les actes avec ses copains. Son entourage a enfin un dos et un fond des yeux qui le contiennent. Par identification, il ressent la sécurité de son dos et de son arrière tête. Son regard s'installe la plupart du temps, mis à part en début de séance quand il est ému de nos retrouvailles. Il cache alors son regard et son sourire dans ses bras quelques instants, en alternant avec un regard lumineux dans ma direction et un grand sourire coquet.

\section{DE LA RÉHUMANISATION D'UN ENTOURAGE CONTENANT À L'IDENTIFICATION À SOI-MÊME}

J'ai été le témoin avec Léo, des étapes de constitution de son moi corporel, qui passe d'abord par l'investissement identificatoire adhésif à l'entourage et par l'annulation de toute ambivalence. L'enfant occupe ainsi les pensées, les émotions et les sensations des autres dans une interchangeabilité des identifications, avant même qu'il puisse s'identifier à lui-même et se glisser dans ses propres ressentis corporels au centre de son entourage dont il vient d'occuper les points de vue. À travers cette interchangeabilité, il passe d'un point de vue à un autre par déplacement, qu'il soit humain, animal, objet animé ou inanimé, ou mouvement et même parfois éclats de lumière, comme on le voit dans les accroches lumineuses ou bien encore sonores, par les rythmes ou la musicalité de la voix. Il réhumanise ainsi son entourage dont il n'a pas encore pu intérioriser les relations qui s'étaient trop vite colorées d'une ambivalence encore inacceptable, avant de s'autoriser à occuper sa place au centre de lui-même.

Il me semble que Léo a dû être confronté dans la première année de sa vie à des investissements ambivalents dus à son handicap, qu'il ne pouvait pas encore intégrer et que son retrait autistique a été un échappatoire de survie psychique où il a tenté de s'autocréer. L'évitement de la relation pouvait être comprise comme une mise en suspens de ses ressentis corporels le temps de son réengendrement. Ainsi en dehors de lui-même, la pensée de son entourage qu'il a dû probablement occuper, a participé à des tentatives de création de scène primitive métaphorisée hors contexte de toute ambivalence, afin de tenter de se créer lui-même dans de meilleures conditions, mais sans y parvenir. Ainsi je comprendrai l'annulation de ses perceptions qu'il a vécue durant de nombreuses années le temps, de son réengendrement psy- 
chique, comme une condition nécessaire pour éviter de contacter des vécus internes incestuels alors qu'il tentait de se réengendrer psychiquement dans une confusion générationnelle. Le retrait dans lequel il s'est placé serait à comprendre comme une barrière anti-incestuelle, au prix d'un effacement de son sentiment d'exister tant qu'il vivait à la place de son entourage, puis d'un effacement de son entourage quand il contactait à nouveau ses ressentis corporels. L'effacement perceptif des temps de passage se traduit chez Léo au moment où il traverse la rue par exemple, où il reste figé sur le passage piéton, ou lorsqu'il met en scène la destruction de toute barrière sur le trajet de retour dans son groupe et au moment d'angoisse à la fin d'une activité, comme si les activités, les lieux, les personnes ou les générations devaient rester clivées pour éviter l'horreur des risques incestuels, de rencontre de ses origines qu'il tente pourtant vainement de réorganiser.

Les séances destructrices du cadre, qu'il a pu vivre pendant de nombreuses semaines au moment de ses débordements émotionnels, s'inscrivent à mon sens dans cette perspective, dans une mise en scène de vécus incestuels dès lors qu'une rencontre avec moi devenait possible mais trop intense dans la réalité de l'espace thérapeutique.

Les séances au ralenti ont permis à mon avis de mettre en place de façon structurante des fantasmes de rencontre de ses origines entre lui et moi, en apaisant les ressentis corporels qu'il le dépassaient. Léo a été protégé des risques incestuels liés à la mise en place de ces fantasmes. Dans un second temps, ces rencontres au ralenti, bien qu'elles aient été peu incarnées, ont constitué une expérience de rencontre avec l'altérité que je représentais. Des ébauches de construction de son moi corporel se sont installées, hors temps et hors champ de l'ambivalence, dans l'acceptation progressive de ses ressentis corporels, sans les effacer ensuite comme il le faisait jusqu'alors pour échapper aux risques de destruction par des vécus incestuels ${ }^{4}$. Son accès à l'ambivalence et à la temporalité ont pu s'élaborer par la suite.

Actuellement Léo progresse bien. Il est davantage investi par les autres dans l'institution et manifeste sa présence et son plaisir à participer aux activités proposées, en particulier à un atelier de conte mimé. Il y tient son rôle dans une véritable précision gestuelle et une certaine grâce corporelle.

J'espère que le tracé de cette rencontre thérapeutique avec Léo pourra éclairer d'autres suivis d'enfants à composantes autistiques, autant que cela l'a été pour moi, afin qu'il puissent assouplir leur barrières autistiques et peut-être les faire céder sans trop de danger, pour accéder à une ambivalence non destructrice.

4. P.-C. Racamier, L'inceste et l'incestuel, Paris, Les Éditions du Collège, 1995, 254 p. 


\section{BIBLIOGRAPHIE}

ANZIEU, D. 1985. Le moi-peau, Paris, Dunod, 254 p.

ANZIEU, D. 1987. "Les signifiants formels et le moi-peau », Les enveloppes psychiques, Paris, Dunod, p. 1-22.

HAAG, G. 1985. « La mère et le bébé dans les deux moitiés du corps », Neuropsychiatrie de l'enfance, 33, p. 107-114.

HAAG, G. 1994. Aux sources de la vie. Le langage préverbal et l'émergence des représentations du corps en situation psychanalytique individuelle ou groupale avec des enfants autistes, "Actes de langage. Quand parler c'est agir », Dialogue, $\mathrm{n}^{\circ} 123$, Paris, $1^{\mathrm{er}}$ trimestre, p. 40-58.

HAAG, G. 1997. Contribution à la compréhension des identifications en jeu dans le moi corporel, dans «Le corps », Journal de psychanalyse de l'enfant, $\mathrm{n}^{\circ}$ 20, Bayard Editions, p. 104-125.

Houzel, D. 1995. « Precipitation anxiety », dans Journal of Child Psychotherapy, vol. 21, 1, p. $65-70$.

Le Poulichet, S. 1994. L'œuvre du temps en psychanalyse, Paris, Rivages, Psychanalyse.

LHEUREUX, C. 2000. L'autisme infantile : angoisses catastrophiques et ébauches d'un contact différencié. Contribution à une clinique des processus psychothérapeutiques, thèse de doctorat de psychopathologie fondamentale et psychanalyse de l'université Paris VII.

Lheureux-DAvidse, C., l'autisme infantile ou le bruit de la rencontre, Paris, L'Harmattan, 2003, $341 \mathrm{p}$.

LHEUREux, C. 2003. «L'image du corps d'une personne abusée dans son enfance », clinique des situations extrêmes, $4^{\mathrm{e}}$ colloque du réseau international européen, Détresse sociale et souffrance psychique, l'enjeu du sujet, 8 au 10 nov. 2000 à Besançon, dans Transumances $n^{\circ} 4$, Presses universitaires de Namur.

Lheureux, C. ; MASSON, C. 2000. «Les fonctions du tracé en thérapie d'enfants. Fonction du dessin dans la rencontre thérapeutique avec un enfant psychotique présentant des retraits autistiques partiels », dans Psychologie clinique, $\mathrm{n}^{\circ} 10$, «Corps, affect, émotion », L'Harmattan, p. 147-166.

Meltzer, D. ; Bremner, J. ; Hoxter, S. ; Weddell, D. 1975. Explorations in Autism, London : the Roland Harris Educational Trust, Clunie Press, trad. fr. G. Haag, Explorations dans le monde de l'autisme : étude psychanalytique, Paris, Payot, coll. «Science de l'homme », 1980, 266 p.

Meltzer, D. ; Williams, M.H. 1988. The Apprehension of beauty. The role of Aesthetic Conflict in Development, Violence and Art, Clunie Press, trad. fr. D. Alcorn, préf. de D. Houzel, L'appréhension de la beauté. Le rôle du conflit esthétique dans le développement Psychique, la Violence, l'Art, éditions du Hublot, coll. «Regards sur les sciences humaines », 2000, 254 p.

RACAMiER, P.-C. 1995. L'inceste et l'incestuel, Paris, Les Éditions du Collège, 254 p.

RaCAMier, P.-C. 1992. Le génie des origines. Psychanalyse et psychoses, Paris, Payot, collection « Bibliothèque scientifique », 420 p. 
SEARles, H. 1979. Contertransference and Related Subjects. Selected Papers, International Universities Press, New York and Mark Paterson, England, trad. fr. de B. Bost : Le contre-transfert, Paris, Gallimard, NRF, coll. "Connaissance de l'inconscient », 1981, 264 p.

Résumé

À partir du matériel clinique du suivi dans un IME d'un jeune atteint de trisomie et présentant des retraits autistiques, je propose de relater quelques séances de la psychothérapie pour repérer les éléments cliniques qui ont permis à Léo de reconstruire peu à peu son image du corps et son sentiment d'exister, et de découvrir un sentiment d'altérité. En quoi la nomination du sensoriel vécu en séance a pu étayer la conscience d'exister de Léo qui se perdait dans un retrait autistique pour survivre, où le clivage dans ses pensées l'empêchait de recontacter son vécu corporel. Les étapes de destructivité (automutilations et violence) peuvent être comprises non pas comme un refus de relation, mais comme des tentatives de différenciation au moment où l'intensité émotionnelle tout juste récupérée le submergeait et que son pare-excitation était encore défaillant. L'intensité émotionnelle le plongeait alors dans des angoisses ingérables de densité de son corps qui ont pu s'apaiser grâce à un ralentissement de vitesse des gestes, des mouvements et des paroles du thérapeute. Ce temps étiré a favorisé l'intégration sensorielle de ses ressentis jusqu'à ce que son image du corps se construise. Ainsi il a goûté peu à peu à la relation à l'autre, dans un premier temps en se mettant dans les pensées d'autrui dans une interchangeabilité d'identifications, pour ensuite s'autoriser à se sentir exister pour lui-même sans s'isoler de la relation. Il a pu ensuite revivre dans le transfert des traumatismes infantiles de s'être senti monstrueux et dangereux pendant la première année de sa vie pour ses parents sous le choc d'avoir et de voir leur bébé marqué d'un handicap. Puis il a pu au fil des séances quitter une responsabilité ingérable de réparer les blessures narcissiques de ses parents pour retrouver enfin sa place dans sa génération et accepter de refaire confiance aux adultes pour se sentir protégé et relancer ses forces de croissance.

Mots-clés

Autisme, handicap, automutilation, image du corps, densité, vitesse, ambivalence.

THE RECONSTRUCTION OF THE BODY IMAGE IN LEO, A CHILD WITH AUTOSOMAL TRISOMY EXHIBITING AUTISTIC WITHDRAWAL

Summary

From clinical material in a Medical-Educational Institution following an adolescent affected by autosomal trisomy and showing signs of autistic withdrawal, I propose to relate several psychotherapeutic sessions in order to trace the clinical elements that allowed Leo to gradually reconstruct his body image and his understanding of existence, and to find a feeling of alterity. As such the naming of sensorial experiences during sessions was able to strengthen Leo's consciousness of his own existence, as 
previously he was losing himself in an autistic withdrawal for his own survival, where the separation of his thoughts kept him from reconnecting with his physical experiences. His stages of destruction (self-abuse and violence) can be understood not as a refusal of relationships, but as attempts at differentiation during moments when a recently recovered emotional intensity overwhelmed him and his pare-excitation was still weak. Emotional intensity thus plunged him into uncontrollable anxiety over the density of his body which could be pacified by a slowing down of gestures and movements, and therapeutic conversations. This expanded time favored the sensorial integration of his feelings until an image of his body could be constructed. Thus he slowly realized relationships with others, at first by thinking of others' identifications as interchangeable, and then by allowing himself to feel his own existence without isolating himself from relationships with others. Next he was able to relive through the transfer of infantile trauma the experience of being seen as monstrous and dangerous during his first year by his parents, who were in shock at seeing their baby marked by a disability. Finally at the end of the sessions he was able to stop an uncontrollable responsibility to repair his parents' narcissistic wounds in order to rediscover his place among his same-age peers, his trust in adults, to feel protected and to begin steps toward further growth.

Keywords

Autism, handicap, self-mutilation, body image, density, speed, ambivalence. 